\title{
Manuelle Lymphdrainage beim Hund
}

\author{
Reinhold Scharwey, Stefanie Süße
}

\author{
Die Manuelle Lymphdrainage ist eine unverzichtbare Behandlungsform zur Therapie von \\ Ödemen. Die Methode ist sehr gut mit Techniken der klassischen Massage, der Manuellen \\ Therapie und der Interferenzstrom-Regulationstherapie kombinierbar. Was muss man über \\ Wirkungsweise, Indikationen und Kontraindikationen der Therapie wissen?
}

\section{Wirkungsweise}

Die Manuelle Lymphdrainage (ML, auch als MLD abgekürzt) ist eine spezielle Massageform z um Abbau von Ödemen und anderen Ablagerungen im Gewebe, z. B. Hämatomen. Als Ödem bezeichnet man eine Ansammlung von Flüssigkeit im Gewebe, die als Schwellung sichtbar wird. Ödeme sind erst ab einer Zunahme der extrazellulären Flüssigkeit von $30 \%$ sichtbar.

\section{Merke \\ Bei Infektionen, toxischen Ödemen und malignen Tumoren ist die Manuelle Lymphdrainage kontraindiziert.}

„Die Manuelle Lymphdrainage nach Vodder beruht auf einer genial erdachten, einfachen und überzeugenden Technik, welche die Beschleunigung des Lymphstroms bewirkt" [1]. Dadurch wird gleichzeitig aber auch ein beruhigender Einfluss auf die sensiblen Nervenendigungen der Haut erreicht. So vermindert sich die Druckschmerzhaftigkeit und es entsteht eine reflektorische Detonisierung des gespannten Gewebes [2].

Die Manuelle Lymphdrainage wirkt während der Druckphase entleerend, in der Entlastungsphase füllend auf die Lymphgefäße. Die Quer- und Längsdehnungen der manuellen Griffe wirken tonisierend auf die glatte Muskulatur und besonders auf die Lymphangien. Als Lymphangion (plural: Lymphangien) bezeichnet man den Abschnitt eines Lymphgefäßes, der zwischen 2 Klappen liegt. Er darf nicht mit einem Lymphangiom, einem benignen Tumor des Lymphgefäßes, verwechselt werden.

Da die Lymphknoten zum Immunsystem gehören, ist ein positiver Effekt auf die Körperabwehr denkbar. (Dr. Emil
Vodder berichtete in den Anfängen über Heilerfolge bei Sinusitis und Schnupfen) [2].

\section{Überblick über die Manuelle Lymphdrainage}

Bereits 1622 zeigte der Italiener Aselli die Lymphgefäße des Darmes eines Hundes. Estrid und Emil Vodder entwickelten erste viele Jahre später die Methode der Manuellen Lymphdrainage in Frankreich (1932-1936). Eine Verbreitung innerhalb Europas über Lehrgänge und Arbeitshefte erfolgte jedoch erst in den 1970er Jahren.

Die Wirkungsweise der Manuellen Therapie war zunächst unklar und beruhte anfangs auf Erfahrungen und Hypothesen. Seit 1973 ist die Manuelle Lymphdrainage als eigenständige Massagetechnik von den Ersatzkassen anerkannt. Bis dahin fehlten die wissenschaftlichen Nachweise, welche vor allem durch Prof. Dr. Michael Földi erbracht wurden [3]. Földi entwickelte die sogenannte komplexe physikalische Entstauungstherapie (KPE).

Diese besteht aus den 4 Elementen:

- Manuelle Lymphdrainage

- Bandagetechniken

- Hautpflege

- Bewegungstherapie

Nach unseren Erfahrungen ist die komplexe physikalische Entstauungstherapie bei Kleintieren nicht gewinnbringend. Bei ihnen ist eine Kombination aus der Manuellen Lymphdrainage, Bewegungstherapie und der Interferenzstrom-Regulationstherapie (IFR) das Mittel der Wahl bei Stauungen.

Im Veterinärbereich ist besonders die Arbeit von Prof. Dirk Berens von Rautenfeld hervorzuheben [4]. 
> Tab. 1 Funktion und Rhythmus der rhythmischen Transportsysteme.

\begin{tabular}{|c|c|c|}
\hline Organsysteme & Hauptfunktionen & Funktionsrhythmen \\
\hline Respirationsapparat & $\begin{array}{l}\text { äußerer Gasstoffwechsel: } \mathrm{O}_{2-} \\
\text { Aufnahme, } \mathrm{CO}_{2} \text {-Abgabe }\end{array}$ & Atemrhythmus: ca. $18 / \mathrm{min}$ \\
\hline Zirkulationsapparat & $\begin{array}{l}\text { - innerer Gasaustausch } \\
\text { - Wärme und Stofftransport } \\
\text { - Aufrechterhaltung des inneren Milieus }\end{array}$ & Pulsfrequenz: ca. $70 / \mathrm{min}$ \\
\hline Lymphsystem/lymphatische Organe & $\begin{array}{l}\text { - Volumenregulation im extrazellulären } \\
\text { Raum } \\
\text { - Abwehr körperfremder Stoffe } \\
\text { - Eiweiß- und Fetttransport } \\
\text { - Aufrechterhaltung der stofflichen } \\
\text { Zusammensetzung der extrazellulären } \\
\text { Flüssigkeit }\end{array}$ & $\begin{array}{l}\text { Kontraktionsfrequenz der Lymphangione: } \\
3-20 / \text { min }\end{array}$ \\
\hline
\end{tabular}

\section{Übersicht der rhythmischen Transportsysteme}

Das Lymphsystem gehört mit in die rhythmischen Transportsysteme des Körpers ( $\triangleright$ Tab.1), die im direkten Zusammenhang mit der inneren Atmung und der Zellernährung stehen.

\section{Anatomie des Lymphsystems}

Das Lymphsystem beginnt blind im Gewebe. Es bildet ein Netz anastomosierender initialer Lymphgefäße und sammelt sich in größeren Lymphgefäßen, die im weiteren Verlauf Lymphknoten ( $\triangleright$ Abb. 1) passieren und in der Nähe des Brusteingangs die Lymphe dem venösen Blut zuleiten. Das heißt, das Lymphsystem endet in dem Venenwinkel zwischen der $\mathrm{V}$. jugularis interna und der V. subclavia jeweils rechts und links kurz vor dem rechten Herzvorhof.

Das Lymphsystem besteht aus den folgenden Gefäßabschnitten:

- initiale Lymphgefäße (Vasa lymphatica)

- Präkollektoren (Vasa lymphatica precollectoria)

- Kollektoren (Vasa collectoria)

- Lymphgefäßstämme (Trunci lymphatici)

- Lymphknoten (Lymphonodi)

\section{Initiale Lymphgefäße}

Sie werden von einschichtigen sich überlappenden Endothelzellen aufgebaut. Die Zellkontaktstrukturen werden von den Zonulae adhaerentes gebildet. Zusätzlich bestehen zwischen den Zellen Kontaktbereiche, die sich ohne Ausbildung der Zonulae adhaerentes lediglich überlappen (Open-Junction-Formationen). Sie bilden die Einlassventile, durch die die extrazelluläre Flüssigkeit in das Röhrensystem gelangt. Erst nach Eintritt in das Röhrensystem wird die extrazelluläre Flüssigkeit als Lymphe bezeichnet. Die Bereitstellung der Open-Junction-Formationen ist ein dynamischer Vorgang, d.h. die Zellen können in Abhängigkeit von mechanischen oder biomechanischen Reizen mehr oder weniger dieser Strukturen zur Verfügung stellen. Die Einlassventile sind durch Ankerfilamente mit dem umgebenden Gewebe verbunden. Durch diese Verbindung muss das Ventil den Bewegungen des Bindegewebes folgen. Zusätzlich bestehen porenförmige Öffnungen, die als Ein- und Auslass dienen.

\section{Präkollektoren}

Die Präkollektoren besitzen bereits Klappen. Die Wände sind 3-schichtig aufgebaut. Es sind Abschnitte mit glatter Muskulatur vorhanden. Es gibt aber auch noch Bereiche mit Einlassfunktion. Die Präkollektoren sammeln die Lymphe aus bestimmten Bereichen des Lymphkapillarnetzes und leiten sie den Lymphkollektoren zu.

\section{Lymphkollektoren}

Es handelt sich um Sammelgefäße (Vasa collectoria). Diese bestehen, ähnlich wie Arterien und Venen, aus 3 Wandschichten: Adventitia, Media und Intima.

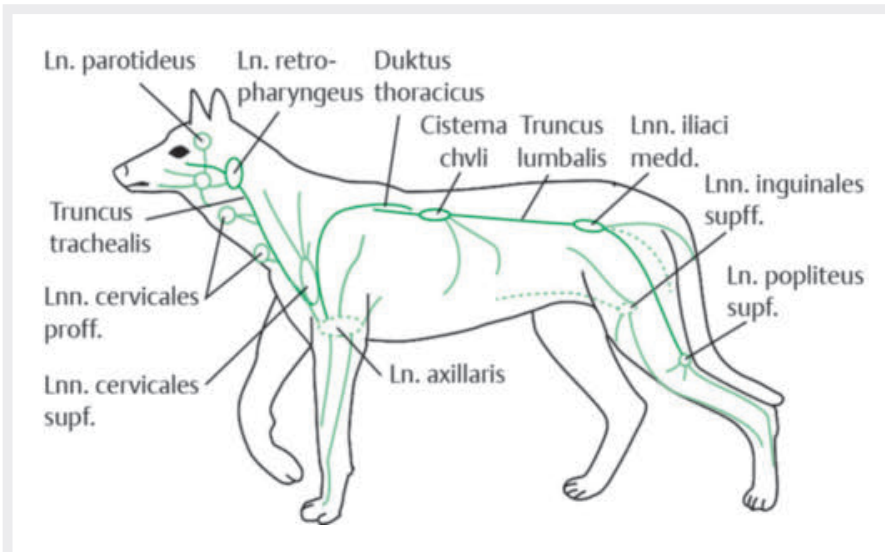

- Abb. 1 Die Lymphknoten beim Hund. Quelle: Thieme, basierend auf Daten von Reinhold Scharwey und nach König/Liebich. Anatomie der Haussäugetiere, Schattauer, 2014 
Oberflächliche Kollektoren, die in der Subkutis liegen, drainieren:

$$
\text { - Haut }
$$

Tiefe Kollektoren, die subfaszial liegen, drainieren:

$$
\begin{aligned}
& \text { - Muskulatur } \\
& \text { - Gelenke } \\
& \text { - Bänder }
\end{aligned}
$$

Oberflächliche und tiefe Lymphkollektoren sind über Anastomosen miteinander verbunden. In die Gefäßwand der Kollektoren sind glatte Muskelzellen eingelagert. Durch Klappen (meist Semilunarklappen) werden die Kollektoren in Segmente unterteilt, sogenannte Lymphangien. Beim Menschen ist die Muskulatur der Lymphangien am deutlichsten ausgebildet. Beim Hund ist sie geringer ausgebildet als beim Menschen.

Die Lymphangien besitzen eine Eigenperistaltik. Sie werden vegetativ über den N. sympathicus gesteuert. Auch ein steigender Fülldruck führt zur Kontraktion ( $\triangleright$ Abb. 2). Skelettmuskulatur, Pulswelle und Atmung können ebenfalls Einfluss auf den Lymphtransport nehmen.

\section{Lymphgefäßstämme}

Die Trunci lymphatici bilden die zentralen Abflusswege für die Lymphe in die großen Körperhöhlen und zum Hals. Letztlich münden sie in die Venenwinkel (Termini).

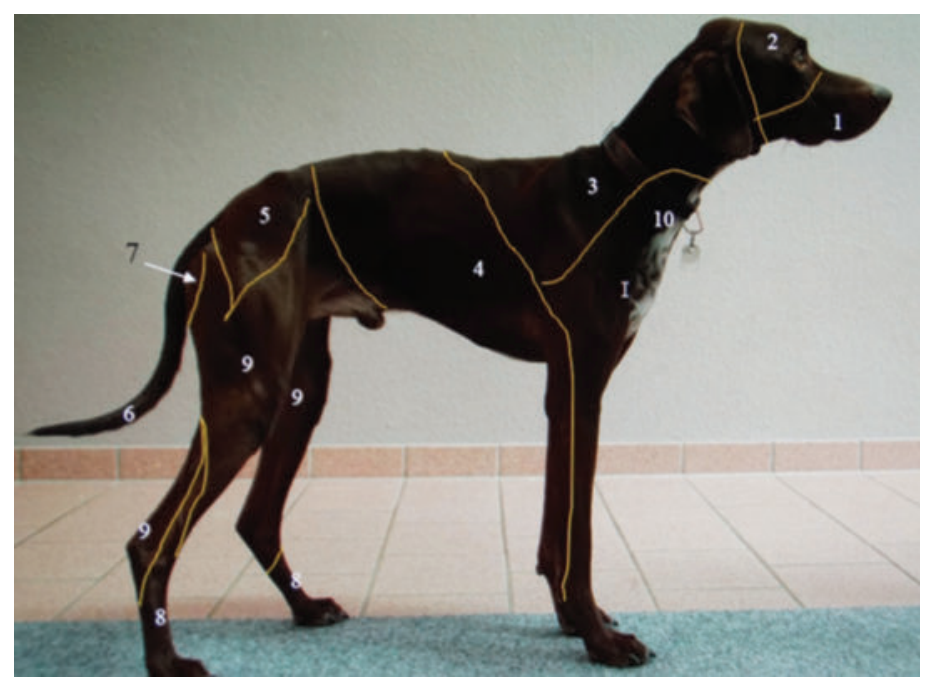

- Abb. 3 Die Entstauung der einzelnen Territorien: Territorium 1 über Lnn. submandibulares, Territorium 2 über die Lnn. parotidei, Territorium 3 über die Lnn. cervicales superficiales, Territorium 4 über die Lnn. axillares, Territorium 5 über die Lnn. iliacales, Territorium 6 über die Lnn. sacrales, Territorium 7 über die Lnn. hypogastrici (Pfeil: Flußrichtung der Lymphe), Territorium 8 über die Lnn. popliteales, Territorium 9 über die Lnn. inguinales, Territorium 10 über die Lnn. cervicales superficiales. Quelle: Reinhold Scharwey basierend auf einer Vorlage von Suami H. et al [5]

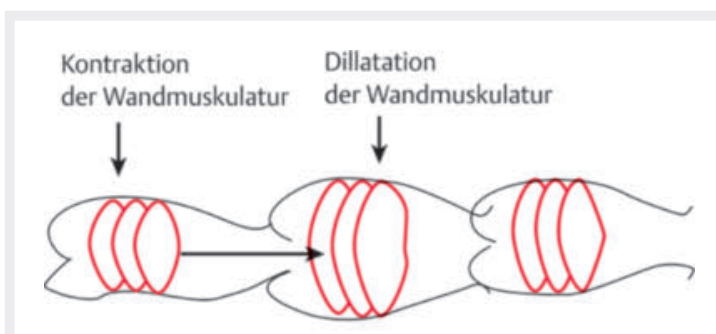

die Lymphe wird aktiv transportiert

- Abb. 2 Die Lymphe wird aktiv durch Kontraktion und Relaxation der Wandmuskulatur transportiert. Quelle: Thieme Gruppe nach einer Vorlage von Reinhold Scharwey

\section{Lymphknoten}

Die Lymphknoten (Lymphonodi: Lnn.) gehören zu den lymphatischen Geweben und sind in den Lymphstrom eingeschaltet. Ihre Größe, Anzahl und Form sind variabel. Sie kommen in Gruppen und als Knotenketten im Bereich der großen Blutgefäße vor. Die Anzahl der Lymphknoten wird beim Hund auf ca. 60 geschätzt [4]. Die Lymphe wird durch die afferenten Lymphgefäße in den Lymphknoten geleitet und durch die efferenten Lymphgefäße abgeleitet.

Der Lymphknoten erfüllt immunologische Aufgaben. $\mathrm{Er}$ ist ein Filter und für bestimmte Stoffe auch ein Depot [4]. Das Blutkapillarnetz des Lymphknotens entzieht der Lymphe Wasser und führt es so dem Blutkreislauf wieder zu.

\section{Lymphscheiden}

Die Hautabschnitte des oberflächlichen Lymphsystems werden je nach Größenordnung der Abflusswege unterteilt:

\section{- Areale \\ - Zonen \\ - Territorien}

Das klappenlose initiale Lymphgefäßnetz überzieht die gesamte Haut. Das initiale Lymphgefäßnetz der Areale wird über Präkollektoren entleert. Mehrere, an einen Kollektor angeschlossene Hautareale, bilden eine Hautzone. Diese sind durch Anastomosen im kutanen Bereich miteinander verbunden. Aber auch Kollaterale benachbarter Kollektoren haben solche Verbindungen. Die Hautzonen, welche von einem Lymphgefäßbündel entsorgt werden, nennt man ein Territorium ( $\triangleright$ Abb.3). Die Grenze zwischen den Territorien nennt man lymphatische Wasserscheide ( $\triangleright$ Abb. 4).

Der ursprüngliche Begriff „lymphatische Wasserscheiden" ist nicht ganz korrekt und wurde nach Grau (1943) in Lymphscheiden umbenannt. Berens von Rautenfeld et al. (1996) schlagen hingegen den Fachterminus Divortia aquorum vor [4]. 


\section{Merke}

Es gibt tierartliche Unterschiede. So sind die Wasserscheiden beim Hund (relative Wasserscheiden) sehr breit und teilen die Körperpartien nur unvollständig. Daher ist es beim Hund leichter, gestaute Bereiche in umgebende Territorien umzuleiten als beim Pferd (s. Artikel in dieser Ausgabe: Manuelle Lymphdrainage beim Pferd).

Der Körper wird durch die Wasserscheiden in eine rechte und linke Hälfte geteilt: die sagittale Wasserscheide.

\section{Physiologie des Lymphsystems}

Die Lymphflüssigkeit (Lymphe) entsteht in dem Moment, in dem die extrazelluläre Flüssigkeit in ein Lymphgefäß einströmt. Sie ist ein Teil des Blutkreislaufs, parallel zum Venensystem.

\section{Merke}

Lymphflüssigkeit ist eine extrazelluläre Flüssigkeit, die von den Lymphkapillaren aufgenommen wurde.

Grundsätzlich besteht zwischen der Filtration und Resorption und der Lymphe ein Gleichgewicht. Die Zuflussmenge entspricht der Abflussmenge. Das Lymphsystem kann unter Umständen ein Vielfaches der normalen Menge transportieren. Es erfüllt damit eine Sicherheitsventilfunktion.

\section{Pathologie des Lymphsystems}

\section{Ödembildung}

Nach Földi werden Störungen im Lymphsystem in 3 Kategorien eingeteilt [3]:

- lymphostatisches Ödem

- lymphodynamisches Ödem

- Sicherheitsventil-Insuffizienz

\section{Lymphostatisches Ödem}

Es ist eiweißreich und entsteht durch mechanische Insuffizienz. Aufgrund organischer oder funktioneller Veränderungen kommt es zum Rückstau im Gewebe. Organische Veränderungen liegen im Bindegewebe. Sie können den Zufluss der prälymphatischen Kanäle behindern. Entwicklungsstörungen können auch die Lymphkapillare betreffen. Verschlüsse der Lymphbahnen können beispielsweise durch Unfälle, Röntgenstrahlen, Operationen oder Tumore entstehen. Funktionelle Störungen können durch gestörte Verschlussmechanismen der Klappen entstehen. Auch Störungen der Lymphangiomotorik sind möglich.

\section{Lymphodynamisches Ödem}

Das lymphodynamische Ödem ist eiweißarm. Es entsteht durch dynamische Insuffizienz der Lymphgefäße. Durch

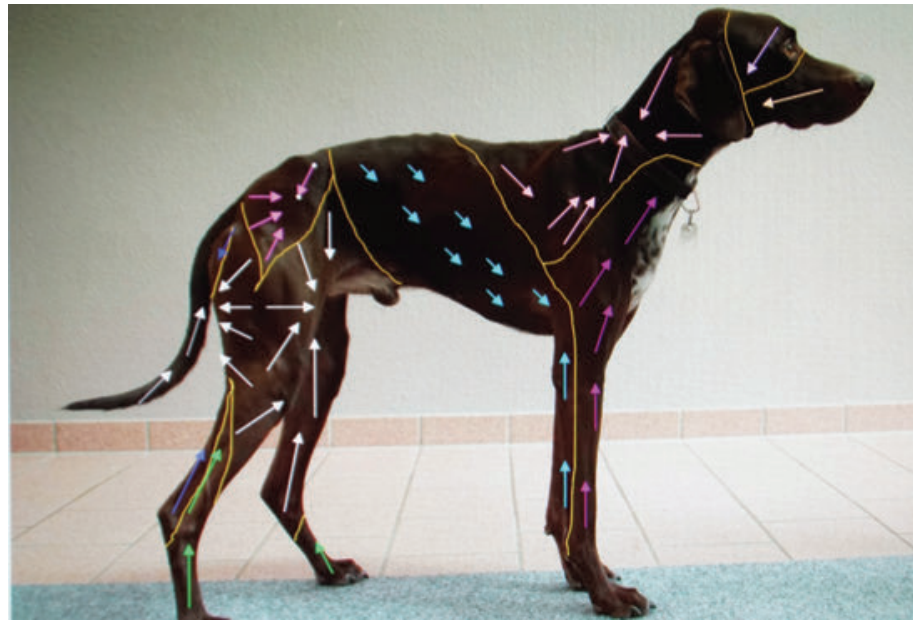

- Abb.4 Die Wasserscheiden des Hundes. Die Pfeile geben die Abflussrichtungen zu den territorialen Lymphknoten an. Quelle: Reinhold Scharwey basierend auf einer Vorlage von Suami H. et al [5]

ein Überangebot an Flüssigkeit kann das Lymphsystem die Flüssigkeitsmenge nicht abtransportieren. Herz- oder Niereninsuffizienzen können der Grund für ein solches Ödem sein. In solchen Fällen ist die Manuelle Lymphdrainage kontraindiziert.

\section{Sicherheitsventil-Insuffizienz}

Sie entsteht, wenn die Transportkapazität des Lymphsystems nicht mehr ausreicht. Es handelt sich um das Zusammentreffen beider Störungsformen, eine gesteigerte lymphatische Last bei absoluter Lymphstauung. Es kommt zu einer qualitativen und quantitativen Stauung, die bis zur Nekrose führen kann.

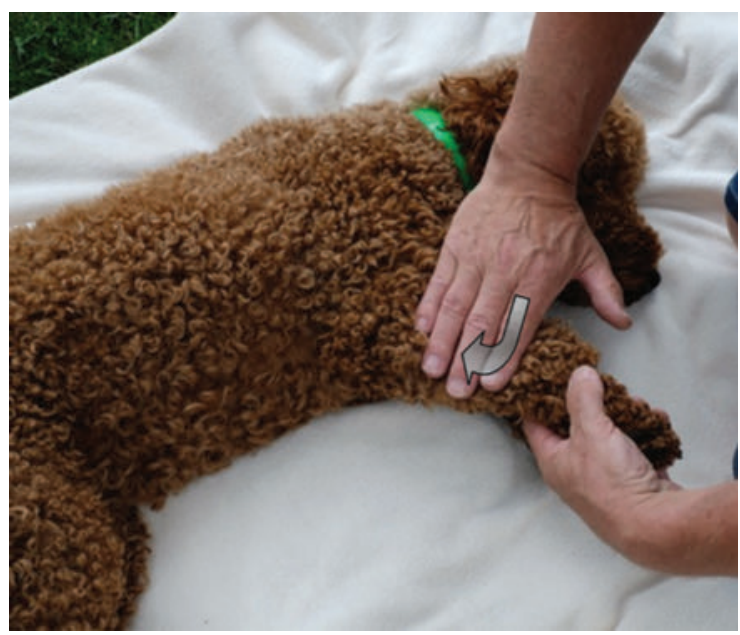

- Abb. 5 Die Finger werden beim stehenden Kreis flach auf das Fell gelegt und die Haut bei sanftem Druck kreisförmig verschoben. Quelle: Reinhold Scharwey 

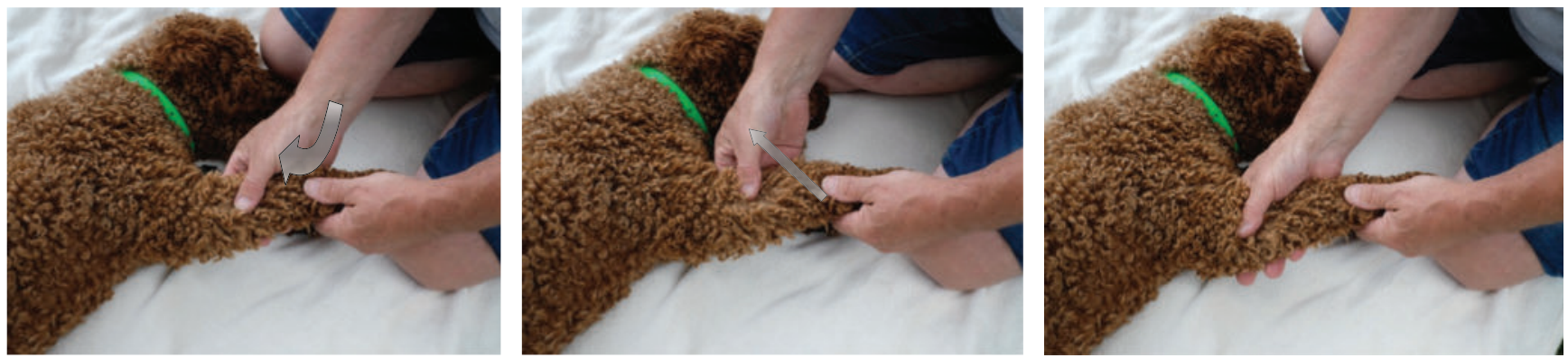

- Abb. 6 Der Daumen wird beim Pumpgriff gegenüber den Fingern positioniert und die Hand abwechselnd angehoben und gesenkt. Die Finger verschieben das Fell dabei quer zum Lymphabfluss. Liegt die Hand dem Fell auf, schiebt sie die Lymphe in Richtung Lymphabfluss bis ans Ende der Dehnfähigkeit, dann gleitet die Hand wieder in ihre Ausgangsposition zurück. Quelle: Reinhold Scharwey.

\section{Manuelle Lymphdrainage}

\section{Indikationen}

Indikationen für die Manuelle Lymphdrainage sind beispielsweise:

- lymphostatische Ödeme (siehe Einteilung nach Földi)

- Traumata: Distorsionen, Kontusionen

- im Rahmen einer Wundbehandlung (auch postoperativ orthopädisch und neurologisch)

- Überlastungssyndrome: Tendinitis, Tendovaginitis, Muskelzerrung/Muskelkater

- Bandscheibenprotusion/-prolaps postoperativ

- Arthrosen, Arthritiden, Spondylarthrosen, Spondylosis deformans

\section{Kontraindikationen}

Man unterscheidet zwischen absoluten und relativen Kontraindikationen für eine manuelle Lymphdrainage.

Absolute Kontraindikationen:

- kardial bedingte Ödeme

- renal bedingte Ödeme

- toxische Ödeme

- alle Arten von Infektionen

- akute Thrombose

- maligne Tumore

Relative Kontraindikationen:

- Hyperthyreose

- Hier verzichtet man auf die Halsbehandlung.

- Halslymphknoten sollten nicht mit Manueller Lymphtherapie behandelt werden, da es zu einer Ausschwemmung der in den Lymphknoten abgekapselten Keime kommen kann.

- Asthma bronchiale

- Während eines Asthmaanfalls soll auf die Manuelle Lymphdrainage verzichtet werden, da sich der Anfall durch den Vagotonus, der durch die Behandlung ausgelöst wird, verstärkt.

- Trächtigkeit
- Aus forensischen Gründen sollten Bauch, Lendenwirbelsäule und hintere Extremitäten in dieser Zeit nicht behandelt werden.

\section{Grundgriffe der Manuellen Lymphdrainage nach Vodder}

\section{Stehender Kreis}

Die Finger werden flach auf das Fell gelegt ( $\triangleright$ Abb.5). Der Druck ist nur so stark, dass die Haut kreisförmig mitverschoben wird. Der Schub geht erst quer zum Lymphabfluss und dann längs. Ist die Grenze der Dehnfähigkeit der Haut erreicht, lässt sich die Hand von der Gewebespannung zurücktragen. In dieser Phase ist der Druck der Finger gleich dem Gewicht einer Fliege. Der Griff kann wiederholt auf der Stelle ausgeführt werden oder spiralförmig in Abflussrichtung.

\section{Pumpgriff}

Die Hand wird diagonal auf die zu behandelnde Extremität gelegt. Der Daumen liegt den Fingern gegenüber ( $\triangleright$ Abb. 6). Die Hand wird nun etwas angehoben, wobei die Finger und der Daumen am Fell bleiben. Dann senkt sich die Hand langsam ab. Die Finger und der Daumen verschieben das Fell nun quer zum Lymphabfluss. Die Handfläche liegt dem Fell jetzt auf und schiebt die Haut in Längsrichtung des Abflusses bis ans Ende der Dehnfähigkeit. Jetzt trägt die Hautspannung die Hand wieder in die Ausgangsposition zurück.

\section{Schöpfgriff}

Die Hand wird diagonal auf die zu behandelnde Extremität gelegt. Der Daumen liegt den Fingern gegenüber $(\checkmark$ Abb. 7). Die Hand wird nun etwas angehoben, wobei Finger und Daumen am Fell bleiben. Jetzt schwingt die Hand mit einer Supinationsbewegung ein. Die Finger nehmen das Fell mit und erzeugen so die Querdehnung im Gewebe. Jetzt senkt sich die Hand ab und der Schub in Längsrichtung erfolgt bis zur Grenze der Dehnfähigkeit. Die Hautspannung trägt die Hand zum Ausgangspunkt zurück. 

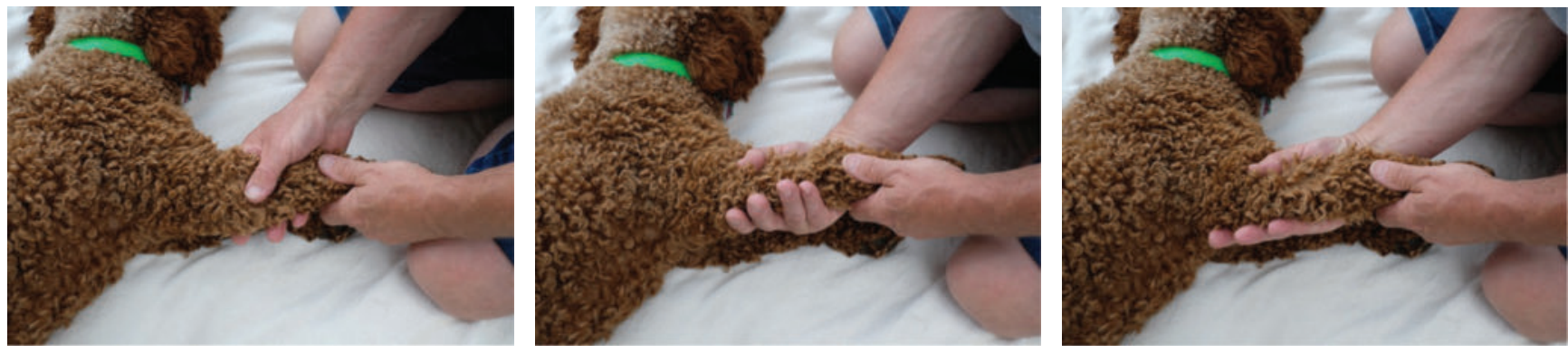

- Abb.7 Der Daumen wird beim Schöpfgriff gegenüber den Fingern positioniert und die Hand leicht angehoben. Die Hand schwingt eine Supinationsbewegung ein, wobei die Finger das Fell mitnehmen und eine Querdehnung im Gewebe erzeugen. Quelle: Reinhold Scharwey.

Auf die Beschreibung des Drehgriffs wird hier verzichtet, da er beim Kleintier kaum Anwendung findet.

\section{Merke}

Je nach Größe des zu behandelnden Tieres können alle Griffe auch mit beiden Händen parallel oder im Wechsel angewendet werden.

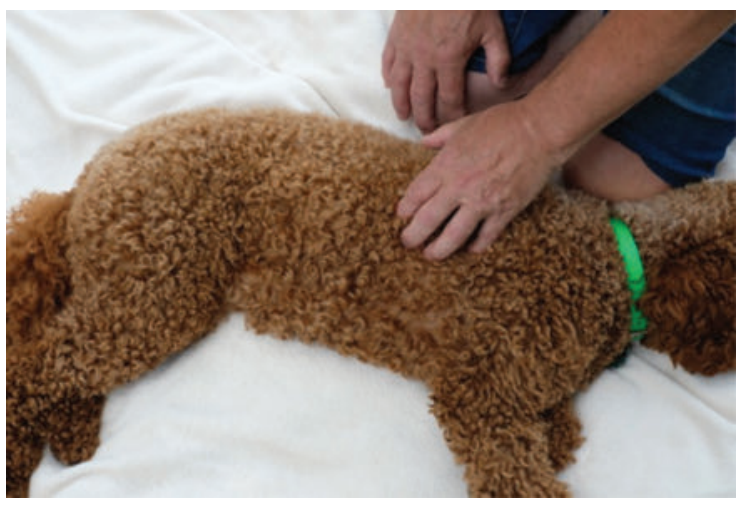

- Abb. 8 Beim Interkostalgriff erzeugen die Fingerspitzen in den Interkostalräumen einen leichten Druck in Richtung Wirbelsäule. Lässt der Druck nach, sinkt die Gewebespannung wieder. Quelle: Reinhold Scharwey

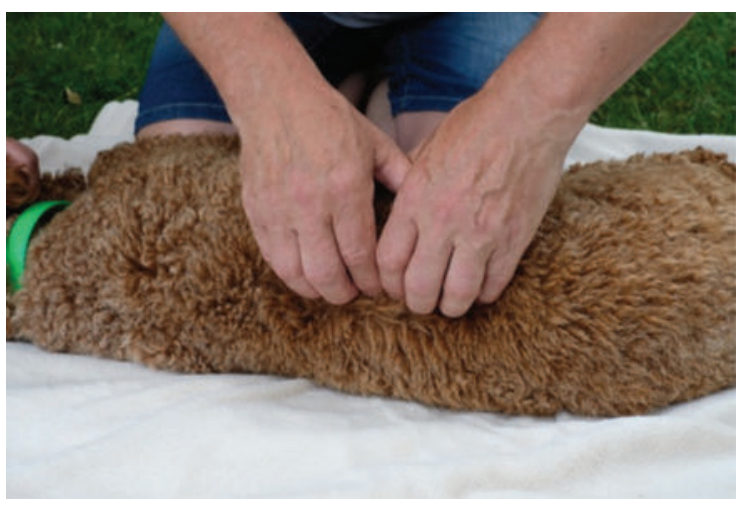

- Abb. 9 Die Fingerspitzen stehen beim Paravertebralgriff steil neben den Dornfortsätzen auf der Rückenmuskulatur. Sie kreisen mit sanftem Druck in Richtung Halswirbelsäule. Das Fell gleitet in der drucklosen Phase wieder in die Ausgangsposition zurück. Quelle: Reinhold Scharwey
Sondergriffe

\section{Interkostalgriff}

Die 4 Fingerspitzen werden in die Interkostalräume gelegt $(\vee$ Abb. 8). Nun wird der Druck sanft in die Tiefe gegeben und in Richtung Wirbelsäule gezogen. Das Fell wird unter den Fingerspitzen mitgenommen. In der drucklosen Phase werden die Finger von der Gewebespannung zurückgetragen.

\section{Paravertebralgriff}

Die 4 Fingerspitzen werden direkt neben den Dornfortsätzen steil auf die Rückenmuskulatur gesetzt ( Abb. 9). Der Druck geht sanft in die Tiefe und es wird in Richtung Halswirbelsäule abgekreist. In der drucklosen Phase trägt das Fell die Fingerspitzen wieder in die Ausgangsstellung zurück.

\section{Pinzettengriff}

Mit gegenübergestelltem Daumen und Zeigefinger können Vorder- und Rückseite kleiner Körperteile gleichzeitig behandelt werden $(\vee$ Abb. 10). Die Haut wird bei sanftem Druck kreisförmig verschoben.

\section{Behandlungsaufbau}

Die Manuelle Lymphdrainage begrenzt die manuelle Druckstärke auf 30-40 Torr. Je nach Tastbefund variiert sie

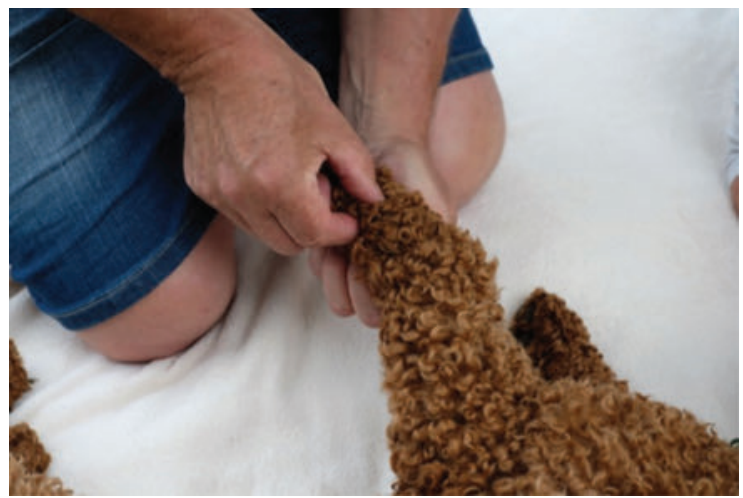

- Abb. 10 Beim Pinzettengriff stehen Daumen und Zeigefinger gegenüber, sodass auch die Gliedmaßen gut therapiert werden können. Der Bewegungsablauf entspricht dem stehenden Kreis. Quelle: Reinhold Scharwey 


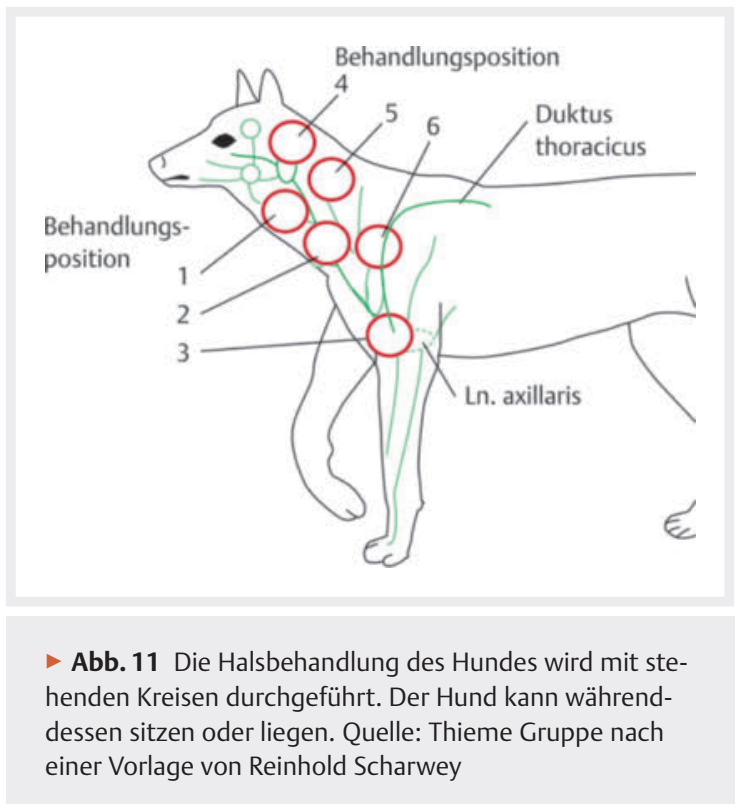

zwischen 0 und 40 Torr. Der optimale Behandlungsdruck ist dem Gewebe angepasst. Da Druckmessungen während der Behandlung nicht möglich sind, ist die Fähigkeit des Therapeuten, die Gewebestruktur zu ertasten, von großer Wichtigkeit. Die Kreisbewegungen werden gleitend stufenlos durchgeführt, um die Quer- und Längsdehnung im Gewebe zu erreichen. Dadurch wird die Lymphangiomotorik erhöht und die Pumpwirkung gewährleistet. Ein gleichmäßiger Rhythmus erhöht die Wirksamkeit. Bei den Griffen und Grifffolgen bleibt der Hautkontakt immer bestehen.

\section{Merke}

Während der gesamten Behandlung muss sich der Therapeut auf die Gewebestruktur konzentrieren. Nur so werden optimale Ergebnisse erreicht.

Es gelten folgende Behandlungsgrundsätze:

- Die Griffe oder Griffvariationen werden 5- bis 7-mal wiederholt, um eine optimale Wirkung zu erzielen.

- Die Druckphase des Griffes dauert länger als die Entspannungsphase.

- Es darf keine Hautrötung entstehen.

- In der Regel wird die Manuelle Lymphdrainage immer mit der Halsbehandlung begonnen.

- An den Extremitäten wird grundsätzlich am proximalen Extremitätenabschnitt mit der Behandlung begonnen.

- Die Behandlungsrichtung ist von distal nach proximal.

- Die Manuelle Lymphdrainage darf niemals Schmerz auslösen.

\section{Halsbehandlung}

- Begonnen und beendet wird immer mit einer Effleurage im Halsbereich.

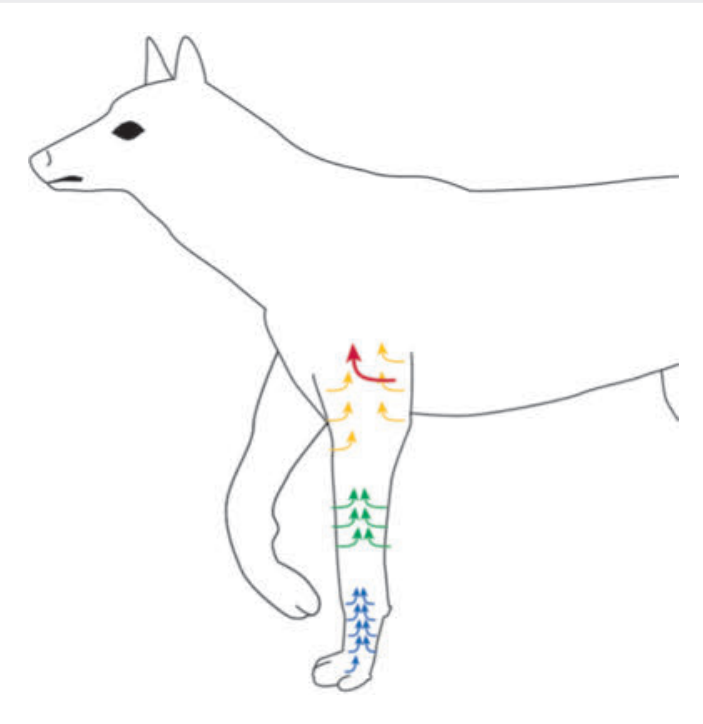

- Abb. 12 Behandlung der Vorderextremität: roter Pfeil: stehender Kreis an der Innenseite; gelbe Pfeile: Pumpgriff am M. triceps und M. triceps brachii; grüne Pfeile: Schöpfgriff an der kranialen und kaudalen Seite; blaue Pfeile: stehende Kreise an der Pfote und an den Zehen. Quelle: Thieme Gruppe nach einer Vorlage Reinhold Scharwey

- Die Behandlungsposition des Tieres kann liegend oder sitzend sein.

- Beide Halsseiten können gleichzeitig behandelt werden.

- Die Halsbehandlung ( $\triangleright$ Abb. 11) wird mit stehenden Kreisen durchgeführt.

- Jede Position wird 5-mal durchgeführt.

- Die Positionen 1-3 werden 3-mal wiederholt, dann werden die Positionen 4-6 3-mal wiederholt.

\section{Behandlung der vorderen Extremität}

- Der Abfluss ist der axilläre Lymphknoten.

- Begonnen wird mit der Effleurage über die gesamte Extremität von proximal nach distal. Zur Anregung des N. sympathicus wird die Extremität von distal nach proximal 4- bis 5-mal mit ganz geringem Kontakt ausgestrichen.

- Im proximalen Bereich des Oberarms wird erst der axilläre Lymphknoten mit stehenden Kreisen angeregt (3-5 Wiederholungen, \ Abb. 12).

- Vom Ellbogen zur Achsel werden auf 2 oder 3 Positionen stehende Kreise durchgeführt (3-5 Wiederholungen).

- Je nach Größe des Tieres folgt jetzt der Pumpgriff über dem M. trapezius und dem M. biceps brachii.

- Der Unterarm kann, je nach Größe des Tieres, von distal nach proximal mit stehenden Kreisen (3-5 Wiederholungen), dem Pumpgriff oder und dem Schöpfgriff behandelt werden. Hier werden 5-7 Wiederholungen durchgeführt. 


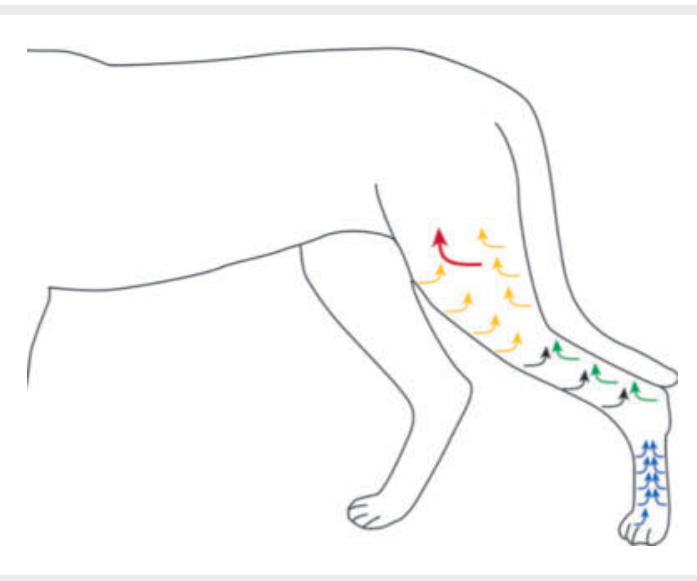

- Abb.13 Behandlung der Hinterextremität: roter Pfeil: stehende Kreise an der Innenseite; gelbe Pfeile kaudal: Pumpgriff über den Hamstrings; gelbe Pfeile kranial: Pumpgriff über dem M. quadriceps femoris; grüne und schwarze Pfeile: Pump- oder Schöpfgriff am Unterschenkel; blaue Pfeile: stehende Kreise an Fuß und Zehen. Quelle: Thieme Gruppe nach einer Vorlage von Reinhold Scharwey

- Die Mittelhand und die Handwurzel werden mit stehenden Kreisen (mit 1 oder 2 Fingern) behandelt.

\section{Behandlung der hinteren Extremität}

- Der Abfluss ist in den Lnn. inguinales superficiales.

- Begonnen wird mit der Effleurage über die gesamte Extremität von proximal nach distal.

Zur Anregung des N. sympathicus (aktiviert die Lymphangiomotorik) wird die Extremität von distal nach proximal 4- bis 5-mal mit ganz geringem Kontakt ausgestrichen.

- Im proximalen Bereich des Oberschenkels werden erst einmal die Lnn. inguinales superficiales mit stehenden Kreisen angeregt (3-5 Wiederholungen,

$\checkmark$ Abb. 13).

- Vom Knie zur Leiste werden auf 2 oder 3 Positionen stehende Kreise durchgeführt (3-5 Wiederholungen).

- Je nach Größe des Tieres folgt jetzt der Pumpgriff über den M. quadriceps femoris und über die Hamstrings (rückseitige Oberschenkelmuskulatur).

- Der Unterschenkel kann, je nach Größe des Tieres, von distal nach proximal mit stehenden Kreisen (3-5 Wiederholungen), dem Pumpgriff oder und dem Schöpfgriff behandelt werden. Hier werden 5-7 Wiederholungen durchgeführt.

- Der Mittelfuß und die Fußwurzel werden mit stehenden Kreisen (mit 1 oder 2 Fingern) behandelt.

- Für die Zehen ist der Pinzettengriff gut geeignet.

\section{Thoraxbehandlung}

- Hier muss zwischen der Behandlung der Haut und der Behandlung der Interkostalmuskulatur unterschieden werden.

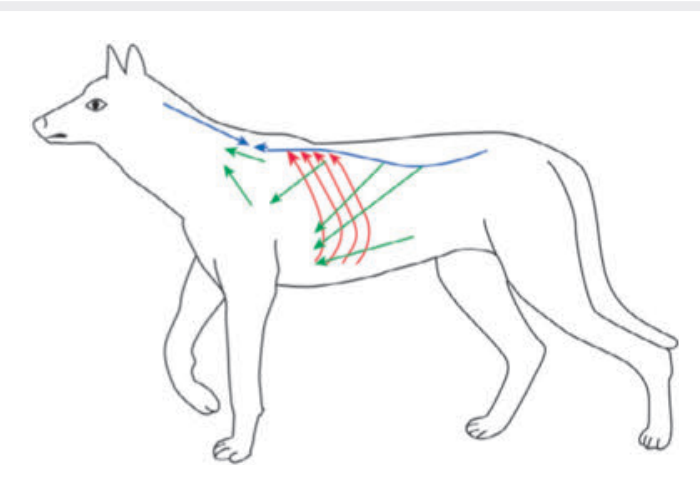

- Abb. 14 Thoraxbehandlung: grüne Pfeile: Behandlung der Haut mit stehenden Kreisen in Richtung Achsel und Halslymphknote; rote Pfeile: Behandlung der Interkostalmuskulatur mithilfe des Interkostalgriffs; blaue Pfeile: Behandlung der Rückenmuskulatur mithilfe des Paravertebralgriffs. Quelle: Thieme Gruppe nach einer Vorlage von Reinhold Scharwey

- Die Haut des Thorax wird in die Lnn. axillares und die Lnn. superficiales cervicales entleert.

- Mit stehenden Kreisen oder dem Drehgriff kann die Haut des Thorax behandelt werden.

- Die Interkostalmuskulatur wird in die paravertebralen Lymphknoten entleert.

- Zur Behandlung der Interkostalmuskulatur ist der Interkostalgriff geeignet.

- Die autochtone Rückenmuskulatur wird mit dem Paravertebralgriff behandelt.

\section{Spezifische Behandlungen}

\section{Kontusion Karpalgelenk}

Je nach Schwere des Traumas ist das Ödem schnell deutlich sichtbar. Als Tierphysiotherapeut arbeitet man verantwortungsvoll nur nach tierärztlicher Diagnosestellung.

- Der kraniale Teil der vorderen Extremität wird über die Lnn. cervicales superficiales entsorgt, der kaudale Anteil über die Lnn. axillares.

- Man beginnt mit der Aktivierung der Lnn. cervicales superficiales. Im Anschluss folgt die Aktivierung der Lnn. axillares.

- Es bietet sich der stehende Kreis als optimaler Griff an.

- Es folgt die Behandlung des proximalen Extremitätenabschnitts beginnend am Ellbogengelenk im kranialen Teil in Richtung Lnn. cervicales ventrales ( $\triangleright$ Abb. 15). Der kaudale Teil wird in Richtung Achsel behandelt.

- Je nach Größe des Tieres bietet sich hier auch der Pump- oder Schöpfgriff an. Auf die gleiche Art wird jetzt der distale Extremitätenabschnitt, proximal der Verletzung beginnend, behandelt.

- Das Karpalgelenk wird, wenn der Schmerz es zulässt, mit stehenden Kreisen proximal des Ödemes beginnend behandelt. 


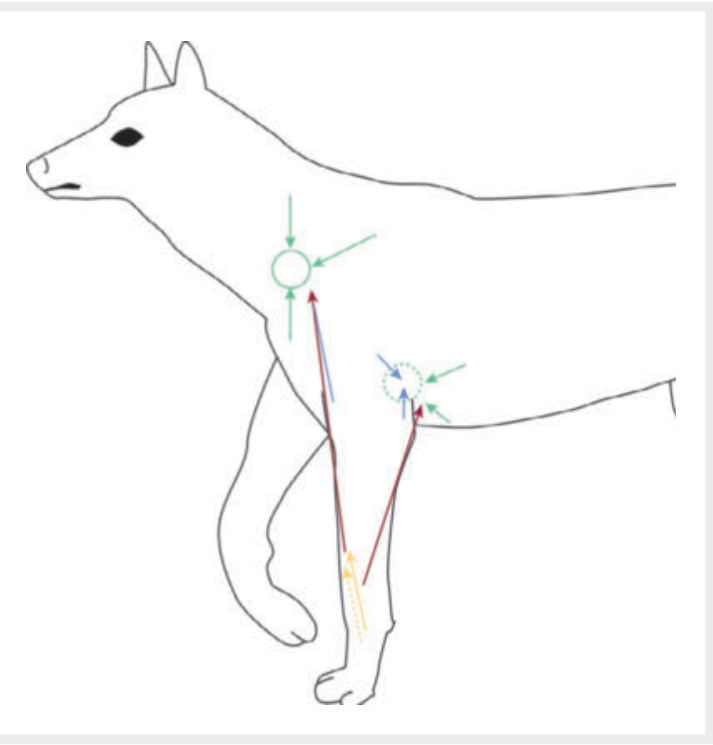

- Abb. 15 Kontusion des Karpalgelenks: grüne Pfeile: Vorbehandlung der Achsel und Halslymphknoten mit stehenden Kreisen; blaue Pfeile: Vorbehandlung der Achsel und Halslymphknoten auf der Innenseite der Extremität mit stehenden Kreisen; rote Pfeile: Behandlung des Brachiums, je nach Größe des Hundes mit dem Pumpgriff oder stehenden Kreisen; gelbe Pfeile: Behandlung des Antibrachiums, je nach Größe des Hundes mit Schöpfgriff, Pumpgriff oder stehenden Kreisen Je kleiner das Tier, umso häufiger wird mit dem stehenden Kreis gearbeitet. Quelle: Thieme Gruppe nach einer Vorlage von Reinhold Scharwey

- Es ist wichtig, den gesamten Ödembereich mit in die Behandlung einzubeziehen, um alle Ablagerungen im Gewebe abzubauen.

\section{Postoperative Wundbehandlung}

Praxisbeispiel Knie-OP links: Der Hund wurde 3 Tage postoperativ zur Behandlung vorgestellt, weil er nur 3 Beine belastete ( $\triangleright$ Abb. 16). Aufgrund der Schmerzhaftigkeit wurden die beiden Lnn. axillares und rechts die Lnn. inguinales behandelt. Schon nach der 1 . Behandlung begann der Hund aufzufußen. Die Behandlung der benachbarten nicht betroffenen Lymphplexus zeigte einen beeindruckenden Erfolg. Das Hämatom konnte durch die 1. Behandlung deutlich verringert werden, dadurch wurde die Wundheilung deutlich beschleunigt.

Tendinitis an der Ursprungssehne des M. biceps brachii In akuten Fällen ist es angeraten, ausschließlich mit der Manuellen Lymphdrainage zu arbeiten. Die Therapie hat durch den Abtransport der Schmerzmediatoren und die Anregung des "Gate-Control-Systems“ eine stark schmerzlindernde Wirkung. Die Reinigung der Sehnenstruktur von Entzündungsmediatoren und die dadurch verbesserte Stoffwechselsituation sind ein weiterer Grund. Es empfiehlt sich eine Vorbehandlung am Hals, um die Lnn. cervicales supferficiales anzuregen. Nun kann die Bizepssehne direkt behandelt werden.

\section{GATE-CONTROL-SYSTEM}

Es handelt sich um eine Mechanorezeption, die im Rückenmark bevorzugt vor der Schmerzrezeption geschaltet wird. Hierdurch kommt es zur Verminderung von Schmerzen.
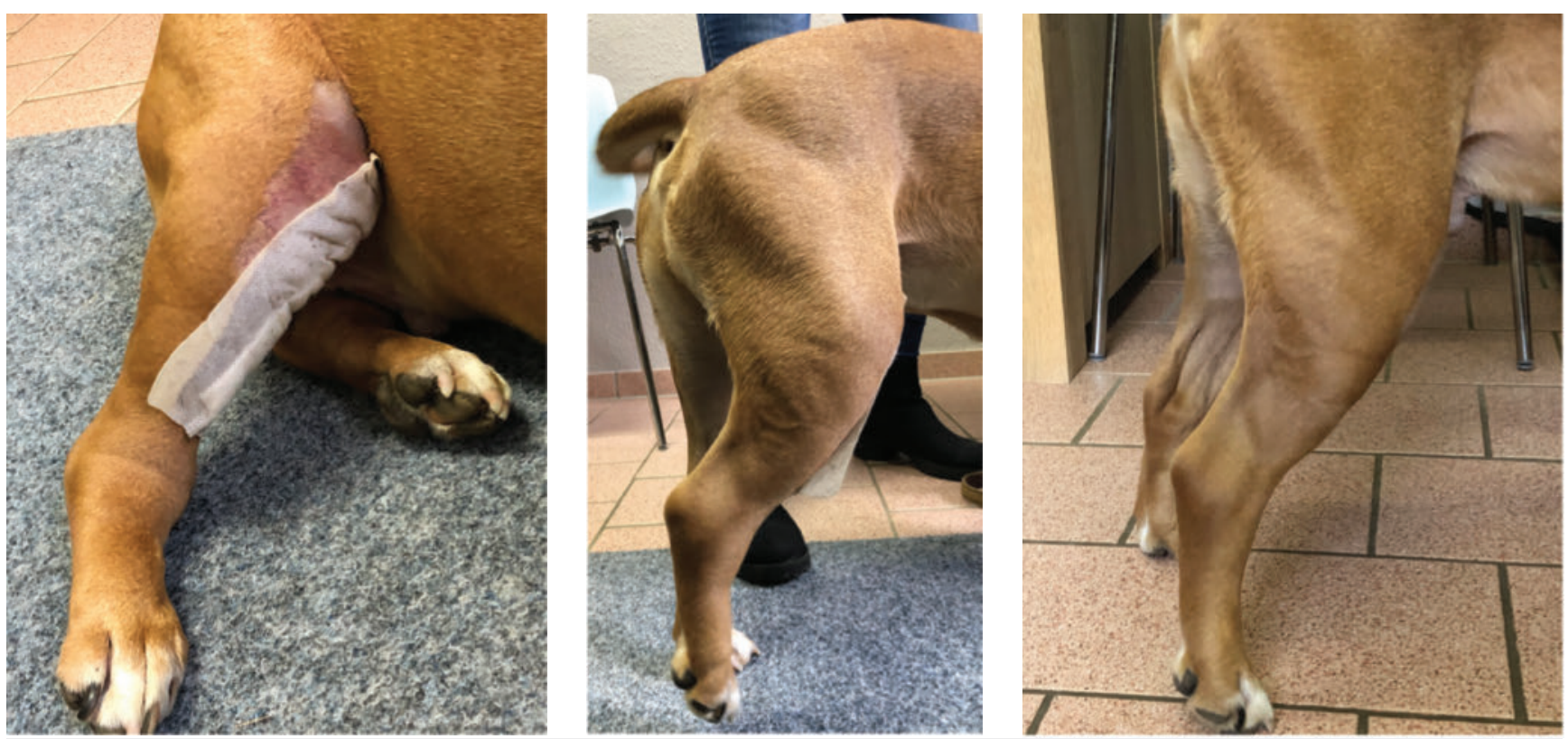

- Abb.16 Der Hund belastete das operierte Bein 3 Tage postoperativ noch nicht. Nach nur einer Anwendung Manueller Lymphdrainage am 5. postoperativen Tag beginnt der Hund die Gliedmaße zu belasten. Quelle: Stefanie Süße und Reinhold Scharwey 


\section{Bandscheibenprolaps postoperativ}

Je nach Lage der OP-Wunde wird das Hautareal in Richtung der zugehörigen Lymphknoten abdrainiert. Kranial der Wunde, im thorakolumbalen Bereich, wird die Rückenmuskulatur mit dem Paravertebralgriff in Richtung Venenwinkel behandelt. Liegt die Wunde im Bereich der Halswirbelsäule wird der Paravertebralgriff kaudal der Wunde beginnend in Richtung Venenwinkel behandelt.

\section{Kombinationsbehandlung}

In chronischen Fällen wird die Manuelle Lymphdrainage durch Querfriktionen an der Sehne ergänzt. Durch den gesteigerten Stoffwechsel in der Sehne lassen sich die Ablagerungen jetzt besser behandeln. Mit dieser Kombination haben die Autoren sehr gute Ergebnisse erreicht.

\section{Merke}

Zur Aufrechterhaltung des Flüssigkeitsgleichgewichts ist es angeraten, nach allen durchblutungsfördernden Maßnahmen eine Manuelle Lymphdrainage durchzuführen.

\section{TAKE HOME}

Die Manuelle Lymphdrainage ist eine unverzichtbare manuelle Behandlungsform in der Physiotherapie. Durch virtuose Anwendungen kann dem Patienten sehr rasch Schmerz genommen werden, zum einen durch das „Gate-Control-System“ und zum anderen durch den beschleunigten Abbau von Schmerz und Entzündungsmediatoren. Die Wundheilung wird durch die Manuelle Lymphdrainage beschleunigt. Entscheidend bei der Manuellen Lymphdrainage ist die Beachtung der Kontraindikationen. Offene Wunden sollten zunächst tierärztlich versorgt werden, da Infektionsgefahr besteht. Als Therapeut muss man immer ganz auf den Patienten konzentriert sein. Routinemäßig durchgeführte Griffe schmälern den Therapieerfolg.

Die Autoren wünschen den Kollegen viel Erfolg bei ihren Therapien.

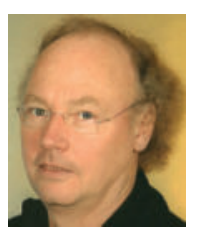

\section{Reinhold Scharwey}

examinierter Masseur, Bademeister und Humanphysiotherapeut; Tierphysiotherapeut (Bad Wildungen); Spezialgebiete: Manuelle Lymphdrainage, neurophysiologische Behandlungverfahren, Elektrotherapie und Interferenzstrom-Regulationstherapie (IFR); seit 2004 Dozent für Physikalische Medizin und Physiotherapie beim Kleintier im Vierbeiner RehaZentrum in Bad Wildungen

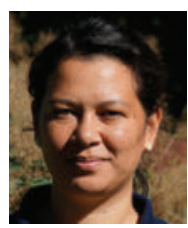

\section{Stefanie Süße}

Tierärztin und Dozentin für Physikalische Medizin und Physiotherapie beim Kleintier im Vierbeiner Reha-Zentrum in Bad Wildungen; Spezialgebiete: Physikalische Medizin und Physiotherapie beim Kleintier, Traditionelle Chinesische Medizin, Goldimplantation und Osteopathie beim Hund; suesse@vierbeinerre hazentrum.de

Literatur

[1] Marnitz H. Ungenutzte Wege der manuellen Behandlung. Heidelberg: Haug; 1981

[2] Wittlinger $\mathrm{H}$, Wittlinger $\mathrm{G}$. Einführung in die Manuelle Lymphdrainage nach Dr. Vodder. Band 1. 8. Aufl. Heidelberg: Haug; 1988

[3] Földi M, Földi E, Kubik S, Hrsg. Lehrbuch Lymphologie. 7. Auff. München: Urban Fischer; 2010

[4] Berens von Rautenfeld D, Hrsg. Manuelle Lymphdrainage beim Pferd. Hannover: Schlütersche; 2005

[5] Suami H, Yamashita S, Soto-Miranda MA et al. Lymphatic territories (lymphosomes) in a canine: an animal model for investigation of postoperative lymphatic alterations. PLoS 2013; 8 (7): e69222

\section{Bibliografie}

DOI https://doi.org/10.1055/a-1046-2296

Hands on 2020; 2: 14-24

(c) Georg Thieme Verlag KG Stuttgart · New York ISSN 2628-6033 


\section{Refresher-Fragen: „Manuelle Lymphdrainage beim Hund“}

Beantworten Sie die folgenden Fragen und prüfen Sie direkt, ob Sie richtig liegen (siehe Seitenrand). Es ist jeweils nur eine Antwort richtig.

Viel Erfolg!

\section{Frage 1}

Das lymphatische System ...

A beschränkt sich auf die Haut.

$B$ beginnt blind in den Geweben und endet in den Venenwinkeln.

C versorgt die Muskulatur mit Nährstoffen.

$\mathrm{D}$ ist direkt an der Blutdruckregulation beteiligt.

\section{Frage 2}

Die Wasserscheiden ...

A trennen den Körper ausschließlich in 2 Hälften.

B stellen Grenzen der arteriellen Blutgefäße dar.

$C$ sind beim Hund relativ angelegt.

D haben keinen Einfluss auf den Behandlungsaufbau.

\section{Frage 3}

Lymphknoten ...

A produzieren die Lymphflüssigkeit.

B sind ein wesentlicher Teil der Immunabwehr.

C sollten vor jeder Manuellen Lymphtherapie ausgedrückt werden.

D haben keinen eigenen Anschluss an das Blutsystem.

\section{Frage 4}

Was versteht man unter der Sicherheitsventilfunktion nach Földi?
A das Öffnen von Hautporen bei starken Ödemen
B dass, die Lymphangione ihre Arbeit um ein Vielfaches steigern können
C die Regulation vom Bluthochdruck
D die Aufnahme von Lymphflüssigkeit durch Venolen

\section{Frage 5}

Der optimale Behandlungsdruck der Manuellen

Lymphdrainage...
A ist nicht wirklich entscheidend.
$B$ ist dem Gewebe angepasst.
C wird regelmäßig gemessen.
D ist abhängig von der Kraft des Therapeuten.

\section{Frage 6}

Bei den Grifftechniken ist es Wichtig, dass die Längsdehnung ...

A Richtung Kopf ausgeführt wird.

B sich an der Fellstruktur orientiert.

C nur im Bereich der Extremitäten wichtig ist.

D sich nach dem Verlauf der Lymphbahnen richtet.

\section{Frage 7}

Welche Indikationen der Manuellen Lymphdrainage gibt es unter anderem?

A Tendinitis, Bandscheibenprolaps postoperativ, kardiales Ödem

B akute Thrombose, Tendovaginitis, lymphostatisches Ödem

C Distorsionen, maligne Tumore, Wundbehandlung postoperativ

D Arthrosen, Kontusionen, Tendinitis, Muskelzerrung

\section{Frage 8}

Wodurch wird die Wirkung der Manuellen Lymphdrainage erreicht?
A intensiver Behandlungsdruck
B sanfte Streichungen
C Quer- und Längsdehnung des Gewebes
D eine Vielzahl von Griffvariationen 